\title{
On finite generation of self-similar groups of finite type
}

\author{
Ievgen V. Bondarenko, Igor O. Samoilovych
}

August 4, 2018

\begin{abstract}
A self-similar group of finite type is the profinite group of all automorphisms of a regular rooted tree that locally around every vertex act as elements of a given finite group of allowed actions. We provide criteria for determining when a self-similar group of finite type is finite, level-transitive, or topologically finitely generated. Using these criteria and GAP computations we show that for the binary alphabet there is no infinite topologically finitely generated self-similar group given by patterns of depth 3 , and there are 32 such groups for depth 4 .
\end{abstract}

Mathematics Subject Classification 2010: 20F65, 20F05, 20E08

Keywords: self-similar group, finite generation, branch group, profinite group

\section{Introduction}

There are two important classes of groups acting on regular rooted trees that have arisen as a generalization of the Grigorchuk group: self-similar groups and branch groups. An automorphism group of a regular rooted tree is self-similar if the restriction of the action of every its element onto every subtree can be given again by an element of the group. There are many examples of self-similar groups with numerous extreme properties (like the Grigorchuk group) and this class of groups is very promising for looking different counterexamples. At the same time, self-similar groups appear naturally in many areas of mathematics and have strong connections with fractal geometry, dynamical systems, automata theory (see [8] and the references therein). Branch groups are automorphism groups of a tree whose subgroup lattice is similar to the tree [1. This class plays an important role in classification of just-infinite groups [5].

Self-similar groups of finite type have arisen as the closure of certain self-similar branch groups in the topology of the tree. It was noticed in [4, Section 7] that the closure of the Grigorchuk group is a profinite self-similar group that can be described by a finite group of allowed local actions on a finite tree (obtained from the binary tree by truncating at some depth). R.I. Grigorchuk used this observation to define a self-similar group of finite type 
as the group of all tree automorphisms that locally around every vertex act as elements of a given finite group (see precise definition in the next section). The term "group of finite type" comes from the analogy with shifts of finite type in symbolic dynamics [7] (note that a different term, namely finitely constrained group, is used in [9, 10]). Every self-similar group of finite type with transitive action on levels of the tree is a profinite branch group by [4, Proposition 7.5], and conversely, the closure (and profinite completion) of a self-similar regular branch group with congruence subgroup property is a self-similar group of finite type by [9, Theorem 3]. The last observation was the main ingredient to compute the Hausdorff dimension of such branch groups in [9].

Although a self-similar group of finite type is easy to define by a finite group of patterns, it is not clear what are the properties of the group. In particular, R.I. Grigorchuk asked in [4, Problem 7.3(i)] under what conditions a self-similar group of finite type is topologically finitely generated. In this note we address this question and establish certain criterion in Theorem 3 as well as some necessary and sufficient conditions. We also answer such basic questions like how to check whether a self-similar group of finite type is trivial, finite, or acts transitively on levels of the tree.

The closure of the Grigorchuk group is a self-similar group of finite type defined by patters of depth 4 over the binary tree. The closure of groups defined in [9] give examples of infinite finitely generated self-similar groups of finite type defined by patterns of depth $d$ for any $d \geq 4$. For depth 2 and binary tree every self-similar group of finite type is either finite or not finitely generated as shown in [10]. The only unknown case was for depth 3. Using developed criteria and GAP computations we prove that there is no infinite finitely generated self-similar group of finite type defined by patterns of depth 3 over the binary tree. For depth 4 there are 32 such groups (including the closures of the Grigorchuk group and the iterated monodromy group of $z^{2}+i[\underline{6}]$ ).

\section{Self-similar groups of finite type}

In this section we first recall all needed definitions and introduce self-similar groups of finite type (see [8, 4] for more information). After that we study conditions when a self-similar group of finite type is trivial, finite, or level-transitive.

Tree $X^{*}$. Let $X$ be a finite alphabet with at least two letters. Let $X^{*}$ be the free monoid freely generated by $X$. The elements of $X^{*}$ are all finite words $x_{1} x_{2} \ldots x_{n}$ over $X$ (including the empty word). We also use notation $X^{*}$ for the tree with the vertex set $X^{*}$ and edges $(v, v x)$ for all $v \in X^{*}$ and $x \in X$. The set $X^{n}$ is the $n$-th level of the tree $X^{*}$. The subtree of $X^{*}$ induced by the set of vertices $\cup_{i=0}^{n} X^{i}$ is denoted by $X^{[n]}$.

Self-similar groups of finite type are defined as special subgroups of the group Aut $X^{*}$ of all automorphisms of the tree $X^{*}$. The group Aut $X^{*}$ is profinite; it is the inverse limit of the sequence

$$
\ldots \rightarrow \text { Aut } X^{[3]} \rightarrow \text { Aut } X^{[2]} \rightarrow \text { Aut } X
$$

where the homomorphisms are given by restriction of the action. 
Sections of automorphisms. For every automorphism $g \in$ Aut $X^{*}$ and every word $v \in X^{*}$ define the section $g_{(v)} \in$ Aut $X^{*}$ of $g$ at $v$ by the rule: $g_{(v)}(x)=y$ for $x, y \in X^{*}$ if and only if $g(v x)=g(v) y$. In other words, the section of $g$ at $v$ is the unique automorphism $g_{(v)}$ of $X^{*}$ such that $g(v x)=g(v) g_{(v)}(x)$ for all $x \in X^{*}$. Sections have the following properties:

$$
g_{(v u)}=g_{(v)(u)}, \quad\left(g^{-1}\right)_{(v)}=\left(g_{\left(g^{-1}(v)\right)}\right)^{-1}, \quad(g h)_{(v)}=g_{(h(v))} h_{(v)}
$$

for all $v, u \in X^{*}$ (we are using left actions, i.e., $\left.(g h)(v)=g(h(v))\right)$.

A subgroup $G<$ Aut $X^{*}$ is called self-similar if $g_{(v)} \in G$ for every $g \in G$ and $v \in X^{*}$.

The restriction of the action of an automorphism $g \in$ Aut $X^{*}$ to the subtree $X^{[d]}$ is denoted by $\left.g\right|_{X^{[d]}} \in$ Aut $X^{[d]}$. To every $g \in$ Aut $X^{*}$ there corresponds a collection $\left(\left.g_{(v)}\right|_{X^{[d]}}\right)_{v \in X^{*}}$ of automorphisms from Aut $X^{[d]}$ which completely describe the action of $g$ on the tree $X^{*}$.

Self-similar groups of finite type. A subgroup $\mathcal{P}$ of Aut $X^{[d]}$ will be called a group of patterns of depth $d$ (or a pattern group of depth $d$ ), $d \geq 1$. We say that an automorphism $g \in$ Aut $X^{*}$ agrees with $\mathcal{P}$ if every section $g_{(v)}, v \in X^{*}$, acts on $X^{[d]}$ in the same way as some element in $\mathcal{P}$, i.e., $\left.g_{(v)}\right|_{X^{[d]}} \in \mathcal{P}$ for all $v \in X^{*}$. Since $\mathcal{P}$ is a group, the inverse $g^{-1}$ and all sections $g_{(v)}$ of such an element $g$ agree with $\mathcal{P}$, the product of two elements that agree with $\mathcal{P}$ also agrees with $\mathcal{P}$. We obtain the self-similar group $G_{\mathcal{P}}$ of all automorphisms $g \in$ Aut $X^{*}$ that agree with $\mathcal{P}$, i.e., we define the group

$$
G_{\mathcal{P}}=\left\{g \in \operatorname{Aut} X^{*}:\left.g_{(v)}\right|_{X^{[d]}} \in \mathcal{P} \text { for every } v \in X^{*}\right\}
$$

called the self-similar group of finite type given by the pattern group $\mathcal{P}$. Note that Grigorchuk in [4] introduced these groups using finite sets of forbidden patterns, while we are using "allowed" patterns.

Every group $G_{\mathcal{P}}$ is closed in the topology of Aut $X^{*}$. Indeed, if for an element $g \in$ Aut $X^{*}$ the restriction $\left.g\right|_{X^{[n]}}$ belongs to $\left.G_{\mathcal{P}}\right|_{X^{[n]}}$ for every $n \in \mathbb{N}$, then $\left.g_{(v)}\right|_{X^{[d]}} \in \mathcal{P}$ for every $v \in X^{*}$ and thus $g \in G_{\mathcal{P}}$. Hence $G_{\mathcal{P}}$ is a profinite group.

Let us consider a few simple examples. If $\mathcal{P}$ is trivial then $G_{\mathcal{P}}$ is trivial. If $\mathcal{P}=$ Aut $X^{[d]}$ then $G_{\mathcal{P}}=$ Aut $X^{*}$ (for any $d \in \mathbb{N}$ ). For every subgroup $\mathcal{P}<\operatorname{Sym}(X)$ the infinitely iterated permutational wreath product $\ldots \imath_{X} \mathcal{P} l_{X} \mathcal{P}$ is a self-similar group of finite type, where $\mathcal{P}$ is the corresponding group of patterns of depth 1 , and every self-similar group of finite type given by patterns of depth 1 is of this form.

Minimal pattern groups. The same self-similar group of finite type may be given by different groups of patterns of depth $d$ and we want to choose a unique pattern group in each class. Let $G$ be a self-similar group of finite type given by a group of patterns of depth $d$ and consider the pattern group $\mathcal{P}=\left.G\right|_{X^{[d]}}$. Since the group $G$ is self-similar, $\left.g_{(v)}\right|_{X^{[d]}} \in \mathcal{P}$ for every $g \in G$ and $v \in X^{*}$, and thus $G<G_{\mathcal{P}}$. On the other hand, it is clear from the definition that every pattern group of depth $d$ that produces $G$ contains $\mathcal{P}$ as a subgroup. Hence $G=G_{\mathcal{P}}$ and $\mathcal{P}$ is the smallest group of patterns of depth $d$ with this property. A pattern group $\mathcal{P}$ of depth $d$ will be called minimal if the equality $G_{\mathcal{P}}=G_{\mathcal{Q}}$ for $\mathcal{Q}<$ Aut $X^{[d]}$ implies $\mathcal{P}<\mathcal{Q}$. It follows from the above arguments that a pattern group $\mathcal{P}$ 
of depth $d$ is minimal if and only if $\mathcal{P}=\left.G_{\mathcal{P}}\right|_{X}[d]$, in other words, if every pattern in $\mathcal{P}$ is realized as a restriction of an element of $G_{\mathcal{P}}$. Every self-similar group of finite type given by patterns of depth $d$ is represented by a unique minimal pattern group of depth $d$.

Pattern graph. Let $\mathcal{P}$ be a group of patterns of depth $d$. In order to minimize $\mathcal{P}$ one can use a directed labeled graph $\Gamma_{\mathcal{P}}$ which we call the pattern graph associated to $\mathcal{P}$. The vertices of $\Gamma_{\mathcal{P}}$ are the elements of $\mathcal{P}$ and for $a, b \in \mathcal{P}$ and $x \in X$ we put a labeled arrow $a \stackrel{x}{\rightarrow} b$ whenever $\left.a_{(x)}\right|_{X^{[d-1]}}=\left.b\right|_{X^{[d-1]}}$. Informally, the arrow $a \stackrel{x}{\rightarrow} b$ shows that we can use the pattern $b$ to extend the action of $a$ on the subtree $x X^{[d]}$ (see Fig. 1). If a vertex $a \in \mathcal{P}$ does not have an outgoing edge labeled by $x$ for some letter $x \in X$, then the action of $a$ cannot be extended to the next level using patterns from $\mathcal{P}$; in other words $a$ is not a restriction of an element of $G_{\mathcal{P}}$. Now it is clear how to minimize $\mathcal{P}$ : we just remove every vertex that does not have an outgoing edge labeled by $x$ for some $x \in X$ and repeat this reduction as long as possible. The remaining patterns will form a minimal pattern group for $G_{\mathcal{P}}$. In particular, $\mathcal{P}$ is minimal if and only if every vertex of $\Gamma_{\mathcal{P}}$ has an outgoing edge labeled by $x$ for every $x \in X$.
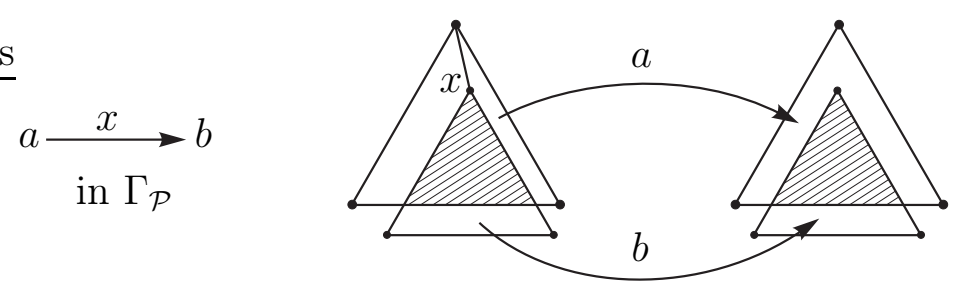

Figure 1: Coordination between patterns.

The graph $\Gamma_{\mathcal{P}}$ can be used to represent elements of the group $G_{\mathcal{P}}$ by graph homomorphisms as follows. Let us take the tree $X^{*}$ and add direction and label to every edge by $v \stackrel{x}{\rightarrow} v x$ for every $v \in X^{*}$ and $x \in X$. Then every element $g \in G_{\mathcal{P}}$ defines a homomorphism $\phi: X^{*} \rightarrow \Gamma_{\mathcal{P}}$ of labeled directed graphs by the rule $\phi(v)=\left.g_{(v)}\right|_{X^{[d]}}$. Indeed, for every arrow $v \stackrel{x}{\rightarrow} v x$ in the tree $X^{*}$ the elements $g_{(v)(x)}$ and $g_{(v x)}$ are the same and we have the arrow $\phi(v) \stackrel{x}{\rightarrow} \phi(v x)$ in the graph $\Gamma_{\mathcal{P}}$. And vise versa, every homomorphism $\phi: X^{*} \rightarrow \Gamma_{\mathcal{P}}$ defines an element $g \in G_{\mathcal{P}}$ by its restrictions $\left.g_{(v)}\right|_{X^{[d]}}=\phi(v)$. This description is an analog of a standard statement in symbolic dynamics that every shift of finite type is sofic (see [7. Theorem 3.1.5]), and pattern graphs play a role of recognition graphs. One can use this observation to introduce the notion of a self-similar group of sofic type which we will discuss elsewhere.

Branching properties. Let us explain the connection between self-similar groups of finite type and branch groups mentioned in Introduction.

Let $G$ be a subgroup of Aut $X^{*}$. The vertex stabilizer $\operatorname{St}_{G}(v)$ of a vertex $v \in X^{*}$ is the subgroup of all $g \in G$ such that $g(v)=v$. The $n$-th level stabilizer $\operatorname{St}_{G}(n)$ is the subgroup of all $g \in G$ such that $g(v)=v$ for every $v \in X^{n}$. Notice that $\operatorname{St}_{G}(v)$ and $\operatorname{St}_{G}(n)$ have finite index in $G$. The rigid vertex stabilizer $\operatorname{RiSt}_{G}(v)$ of a vertex $v \in X^{*}$ is the subgroup 
of all $g \in G$ such that $g(u)=u$ for every vertex $u \in X^{*} \backslash v X^{*}$. The set of all sections $g_{(v)}$ for $g \in \operatorname{RiSt}_{G}(v)$ forms a group which we call the section group of $\operatorname{RiSt}_{G}(v)$ at the vertex $v$. The group $G$ is called level-transitive if it acts transitively on all levels $X^{n}$ of the tree. The group $G$ is called regular branch branching over its subgroup $K$ if $G$ is level-transitive, $K$ is a normal subgroup of finite index, and the group of all automorphism $g \in \operatorname{St}_{\text {Aut } X^{*}}(1)$ such that the tuple $\left(g_{(x)}\right)_{x \in X}$ belongs to $\prod_{X} K$ is a subgroup of finite index in $K$. Note that the last condition implies that the section group of $\operatorname{RiSt}_{K}(v)$ at $v$ contains $K$ for every vertex $v \in X^{*}$.

Every level-transitive self-similar group $G_{\mathcal{P}}$ of finite type given by patterns of depth $d$ is regular branch over its level stabilizer $\operatorname{St}_{G_{\mathcal{P}}}(d-1)$ (see [4, Proposition 7.15]). Indeed, notice that for every element $h \in \mathrm{St}_{G_{\mathcal{P}}}(d-1)$ and any vertex $v \in X^{*}$ the unique automorphism $g \in \operatorname{RiSt}_{\text {Aut } X^{*}}(v)$ such that $g_{(v)}=h$ agrees with the pattern group $\mathcal{P}$ and hence belongs to $G_{\mathcal{P}}$. It follows that $\operatorname{St}_{G_{\mathcal{P}}}(n)$ for $n \geq d$ decomposes into the direct product

$$
\operatorname{St}_{G_{\mathcal{P}}}(n) \cong \operatorname{St}_{G_{\mathcal{P}}}(d-1) \times \ldots \times \operatorname{St}_{G_{\mathcal{P}}}(d-1)
$$

of $|X|^{n-d+1}$ copies of $\operatorname{St}_{G_{\mathcal{P}}}(d-1)$, where each factor acts on the corresponding subtree $v X^{*}$ for $v \in X^{n-d+1}$. The last condition in the definition of a regular branch group follows. Conversely, if $G$ is a self-similar regular branch group branching over its level stabilizer $\mathrm{St}_{G}(d-1)$ then the closure of $G$ in Aut $X^{*}$ is a self-similar group of finite type given by patterns of depth $d$ (see [9, Theorem 3]).

Triviality, finiteness, and level-transitivity of $G_{\mathcal{P}}$. Given a pattern group $\mathcal{P}$ we want to understand whether the group $G_{\mathcal{P}}$ is trivial, finite, or acts transitively on the levels of the tree $X^{*}$. The answer to the question about triviality of $G_{\mathcal{P}}$ directly follows from the definition of a minimal pattern group. Namely, the group $G_{\mathcal{P}}$ is trivial if and only if minimizing $\mathcal{P}$ we obtain the trivial group.

The finiteness of $G_{\mathcal{P}}$ can be effectively checked using the next statement.

Proposition 1. Let $\mathcal{P}$ be a minimal pattern group of depth $d$. The group $G_{\mathcal{P}}$ is finite if and only if the stabilizer $\operatorname{St}_{\mathcal{P}}(d-1)$ is trivial, and in this case $G_{\mathcal{P}}$ is isomorphic to $\mathcal{P}$.

Proof. Let $\Gamma_{\mathcal{P}}$ be the pattern graph of $\mathcal{P}$ and put $m=\left|\operatorname{St}_{\mathcal{P}}(d-1)\right|$. Notice that $\left.(b c)\right|_{X^{[d-1]}}=$ $\left.b\right|_{X^{[d-1]}}$ for every $b \in \mathcal{P}$ and $c \in \operatorname{St}_{\mathcal{P}}(d-1)$. Hence if $a \stackrel{x}{\rightarrow} b$ is an arrow in $\Gamma_{\mathcal{P}}$ then $a \stackrel{x}{\rightarrow} b c$ is also an arrow in $\Gamma_{\mathcal{P}}$ for every $c \in \operatorname{St}_{\mathcal{P}}(d-1)$, and every outgoing arrow at $a$ with label $x$ is of this form. Therefore, since $\mathcal{P}$ is minimal, every vertex of $\Gamma_{\mathcal{P}}$ has precisely $m$ outgoing edges labeled by $x$ for every $x \in X$. It follows that for every $a \in \mathcal{P}$ there are precisely $m^{|X|}$ elements $\left.g \in G_{\mathcal{P}}\right|_{X^{[d+1]}}$ such that $\left.g\right|_{X^{[d]}}=a$; in other words, every pattern in $\mathcal{P}$ has $m^{|X|}$ extensions to the next level. Then for each level $n>d$ and for every $\left.f \in G_{\mathcal{P}}\right|_{X^{[n]}}$ there are precisely $m^{|X|^{n-d+1}}$ elements $\left.g \in G_{\mathcal{P}}\right|_{X^{[n+1]}}$ such that $\left.g\right|_{X^{[n]}}=f$. Now we can compute the total number of elements in the restriction $\left.G_{\mathcal{P}}\right|_{X[n]}$ :

$$
\left|G_{\mathcal{P}}\right|_{X[n]}|=| \mathcal{P} \mid \cdot m^{|X|+|X|^{2}+\ldots+|X|^{n-d}}, \text { for } n>d .
$$

Therefore the group $G_{\mathcal{P}}$ is finite if and only if $m=1$, i.e., when the $\operatorname{group} \operatorname{St}_{\mathcal{P}}(d-1)$ is trivial. In this case, $\left|G_{\mathcal{P}}\right|=|\mathcal{P}|$ and the restriction $\left.g \mapsto g\right|_{X^{[d]}}$ is an isomorphism between $G_{\mathcal{P}}$ and $\mathcal{P}$. 
It follows from the proof that we can also use the pattern graph $\Gamma_{\mathcal{P}}$ to check the finiteness of $G_{\mathcal{P}}$. If $\mathcal{P}$ is minimal, then the group $G_{\mathcal{P}}$ is finite if and only if some (equivalently, every) vertex of $\Gamma_{\mathcal{P}}$ has only one outgoing edge labeled by $x$ for each $x \in X$.

Let us treat transitivity on levels. We will use the standard observation that a subgroup $G<$ Aut $X^{*}$ acts transitively on $X^{n+1}$ if and only if it acts transitively on $X^{n}$ and the stabilizer $\operatorname{St}_{G}(v)$ of some (every) vertex $v \in X^{n}$ acts transitively on $v X$.

Let $\mathcal{P}$ be a minimal pattern group of depth $d$ and consider the self-similar group of finite type $G_{\mathcal{P}}$. We fix a letter $x \in X$ and use notation $x^{n}$ for the word $x \ldots x$ ( $n$ times). Let $\mathcal{P}_{n}$ be the group of all elements $a \in \mathcal{P}$ for which there exists $g \in \operatorname{St}_{G_{\mathcal{P}}}\left(x^{n}\right)$ such that $\left.g_{\left(x^{n}\right)}\right|_{X^{[d]}}=a$. Then $\operatorname{St}_{G_{\mathcal{P}}}\left(x^{n}\right)$ is transitive on $x^{n} X$ if and only if $\mathcal{P}_{n}$ is transitive on $X$. It follows that $G_{\mathcal{P}}$ is level-transitive if and only if each group $\mathcal{P}_{n}$ for $n \geq 0$ is transitive on $X$. Notice that the groups $\mathcal{P}_{n}$ can be computed recursively by the rule: $\mathcal{P}_{0}=\mathcal{P}$ and

$$
\mathcal{P}_{n+1}=\left\{a \in \mathcal{P}_{n}: \text { there exists } b \in \operatorname{St}_{\mathcal{P}_{n}}(x) \text { such that }\left.b_{(x)}\right|_{X^{[d-1]}}=\left.a\right|_{X^{[d-1]}}\right\} .
$$

We obtain a decreasing sequence $\mathcal{P}>\mathcal{P}_{1}>\ldots$ of finite groups which should stabilize on some subgroup $\mathcal{Q}<\mathcal{P}, \mathcal{Q}=\cap_{n \geq 0} \mathcal{P}_{n}$. Moreover, if we take the smallest $n$ such that $\mathcal{P}_{n}=\mathcal{P}_{n+1}$ then $\mathcal{P}_{n}=\mathcal{P}_{n+k}$ for every $k \in \mathbb{N}$, and thus $\mathcal{Q}=\mathcal{P}_{n}$. Hence the group $\mathcal{Q}$ can be algorithmically computed. We have proved the following effective criterium.

Proposition 2. The group $G_{\mathcal{P}}$ is level-transitive if and only if the group $\mathcal{Q}$ is transitive on $X$.

\section{$3 \quad$ Finite generation of groups $G_{\mathcal{P}}$}

In this section we study when the group $G_{\mathcal{P}}$ is topologically finitely generated. Further we omit the word "topologically".

Theorem 3. Let $G$ be a level-transitive self-similar group of finite type given by patterns of depth $d$. The group $G$ is finitely generated if and only if there exists $n \geq d$ such that the commutator of $\left.\operatorname{St}_{G}(d-1)\right|_{X^{[n]}}$ contains $\left.\operatorname{St}_{G}(n-1)\right|_{X^{[n]}}$.

Proof. Let $G=G_{\mathcal{P}}$ for a minimal pattern group $\mathcal{P}$ of depth $d$.

First we prove the necessity. The proof will not use transitivity on levels. Assume that the commutator of $\left.\operatorname{St}_{G}(d-1)\right|_{X^{[n]}}$ does not contain $\left.\operatorname{St}_{G}(n-1)\right|_{X^{[n]}}$ for every $n \geq d$. Let us prove that $\operatorname{St}_{G}(d-1)$ and thus $G$ are not finitely generated. In the proof we will use notations $S=\operatorname{St}_{G}(d-1)$ and $S_{n}=\left.S\right|_{X}[n]$. For each $m \geq d$ consider the homomorphism

$$
\varphi: S \rightarrow \prod_{n=d}^{m} S_{n} /\left[S_{n}, S_{n}\right], \varphi(g)=\left(\left.g\right|_{X^{[n]}}\left[S_{n}, S_{n}\right]\right)_{n=d}^{m} .
$$

Recall that the stabilizer $\left.\operatorname{St}_{G}(n-1)\right|_{X}{ }^{[n]}$ decomposes into the direct product $\operatorname{St}_{\mathcal{P}}(d-1) \times$ $\ldots \times \operatorname{St}_{\mathcal{P}}(d-1)$ of $|X|^{n-d}$ copies of $\operatorname{St}_{\mathcal{P}}(d-1)$. By our assumption there exists an element $g_{n}=\left(1, \ldots, a_{n}, \ldots, 1\right), a_{n} \in \operatorname{St}_{\mathcal{P}}(d-1)$, of this product that does not belong to the 

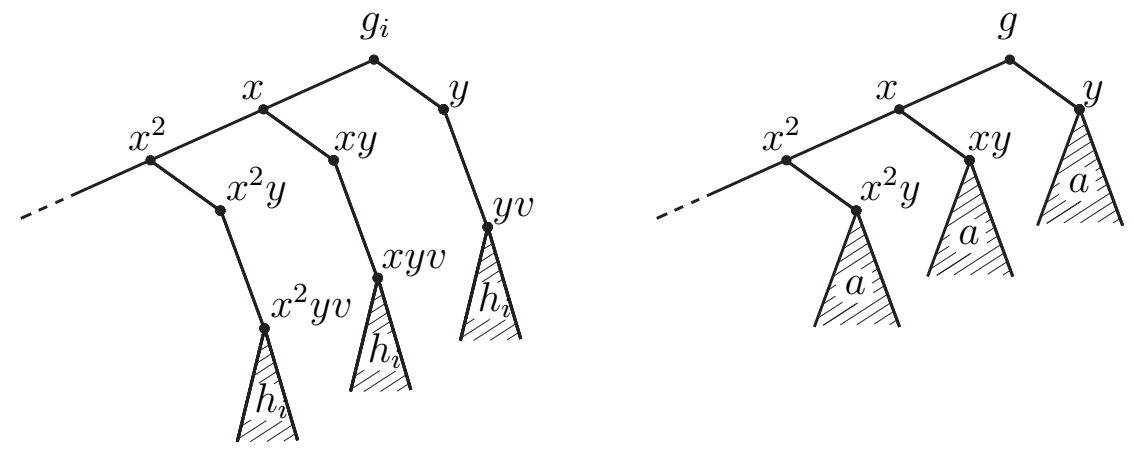

Figure 2: The construction of generator $g_{i}$ and conjugator $g$.

commutator $\left[S_{n}, S_{n}\right]$. Let $A_{n}$ be the group generated by the image of $g_{n}$ in the quotient of $\left.\mathrm{St}_{G}(n-1)\right|_{X^{[n]}}$ by $\left[S_{n}, S_{n}\right]$. The group $A_{n}$ is a nontrivial subgroup of the finite abelian group $S_{n} /\left[S_{n}, S_{n}\right]$. Hence $A_{n}$ is also a quotient of $S_{n} /\left[S_{n}, S_{n}\right]$. Composing with $\varphi$ we obtain a homomorphism from $S$ to $\prod_{n=d}^{m} A_{n}$. Moreover, for $i<n$ the $i$-th component of the image of $g_{n}$ in this direct product is trivial. It follows that $\prod_{n=d}^{m} A_{n}$ is a homomorphic image of $S$. Since $\left|A_{n}\right| \leq|\mathcal{P}|$ for all $n$, the number of generators of $\prod_{n=d}^{m} A_{n}$ goes to infinity as $m$ goes to infinity. Hence $S$ is not finitely generated.

Let us prove the converse. Fix $k \geq d$ such that the commutator of $\left.\operatorname{St}_{G}(d-1)\right|_{X^{[k]}}$ contains $\left.\operatorname{St}_{G}(k-1)\right|_{X[k]}$. We construct a finitely generated dense subgroup of $G$ using the techniques from branch groups (see [1, 2]). Let $f_{1}, \ldots, f_{l}$ and $h_{1}, \ldots, h_{m}$ be the elements of $G$ such that

$$
\left.\left\langle f_{1}, \ldots, f_{l}\right\rangle\right|_{X^{[1+d+k]}}=\left.G\right|_{X^{[1+d+k]}} \text { and }\left.\left\langle h_{1}, \ldots, h_{m}\right\rangle\right|_{X^{[k]}}=\left.\operatorname{St}_{G}(d-1)\right|_{X^{[k]}} .
$$

The group $\operatorname{St}_{\mathcal{P}}(d-1)$ is nontrivial by Proposition 1, and we can find $v \in X^{d}$ and $a \in$ $\mathrm{St}_{G}(d-1)$ such that $a(v) \neq v$ (the element $a$ will be used to shift the section of certain automorphisms at the vertex $v$ ). Fix two letter $x, y \in X, x \neq y$. Define the automorphisms $g_{1}, \ldots, g_{m}$ recursively by their sections:

$$
g_{i(y v)}=h_{i} \text { and } g_{i(x)}=g_{i}, i=1, \ldots, m,
$$

and the other sections are trivial (see Fig. 2). Notice that $g_{1}, \ldots, g_{m}$ belong to $G$.

Consider the group $H=\left\langle f_{1}, \ldots, f_{l}, h_{1}, \ldots, h_{m}, g_{1}, \ldots, g_{m}\right\rangle$ and let us show that $H$ is dense in $G$. We need to prove that $\left.H\right|_{X^{[n]}}=\left.G\right|_{X^{[n]}}$ for all $n \in \mathbb{N}$. The statement holds for $n \leq 1+d+k$ by construction. By induction on $n$ assume that we have proved it for all levels $\leq n+d+k$. There exists an element $g \in G$ such that

$$
g_{\left(x^{i} y\right)}=a \text { for } i=0, \ldots, n-1
$$

and the other sections are trivial (see Fig. 2). By inductive hypothesis there exists $h \in H$ such that $\left.h\right|_{X^{[n+d+k]}}=\left.g\right|_{X^{[n+d+k]}}$. Then the commutator $\left[h^{-1} g_{i} h, g_{j}\right]$ acts trivially on the vertices in $X^{[n+d+k+1]} \backslash x^{n} y v X^{[k]}$ and at the vertex $x^{n} y v$ has section

$$
\left[h^{-1} g_{i} h, g_{j}\right]_{\left(x^{n} y v\right)}=\left[h_{\left(x^{n} y v\right)}^{-1} h_{i} h_{\left(x^{n} y v\right)}, h_{j}\right] .
$$


Conjugating by generators $g_{1}, \ldots, g_{m}$ we obtain that the section group of $\left.\operatorname{RiSt}_{H}\left(x^{n} y v\right)\right|_{X^{[n+d+k+1]}}$ at $x^{n} y v$ contains the commutator of $\left.\operatorname{St}_{G}(d-1)\right|_{X^{[k]}}$ and hence $\left.\operatorname{St}_{G}(k-1)\right|_{X^{[k]}}$. Since the action is transitive this holds for every vertex of the level $X^{n+d+1}$. Hence $\operatorname{St}_{H}(n+d+$ $k)\left.\right|_{X^{[n+d+k+1]}}=\left.\operatorname{St}_{G}(n+d+k)\right|_{X^{[n+d+k+1]}}$ and the statement follows.

Remark 1. An automorphism of the tree $X^{*}$ is called finite-state if it has finitely many sections (the term comes from automata theory); a subgroup is finite-state if it consists of finite-state automorphisms. We can always choose elements $f_{1}, \ldots, f_{l}$ and $h_{1}, \ldots, h_{m}$ so that they are finite-state. Then the elements $g_{1}, \ldots, g_{m}$ and the group $H$ constructed in the proof will be also finite-state. Adding sections of elements we obtain a finitely generated finite-state self-similar dense subgroup in $G$.

Remark 2. The condition of level-transitivity cannot be dropped in Theorem [3. For example, consider the alternating group $A_{5}$ with the natural action on $\{1,2,3,4,5\}$, extend the action to the alphabet $X=\{0,1,2,3,4,5\}$ by putting $\pi(0)=0$ for every $\pi \in A_{5}$, and consider the infinitely iterated permutational wreath product $G_{A_{5}}=\ldots \imath_{X} A_{5} \imath_{X} A_{5}$. The group $A_{5}$ is perfect, i.e., $\left[A_{5}, A_{5}\right]=A_{5}$, hence the condition in Theorem 3 holds for $n=$ $d=1$. However the group $G$ is not finitely generated, because the map $g \mapsto\left(\left.g_{\left(0^{n}\right)}\right|_{X}\right)_{n \in \mathbb{N}}$ is a surjective homomorphism from $G$ to the product $\prod_{\mathbb{N}} A_{5}$ which is not finitely generated.

Remark 3. It is not difficult to see that for a group $G_{\mathcal{P}}$ given by a transitive pattern group $\mathcal{P}$ of depth 1 the condition in the theorem holds for some $n$ if and only if the group $\mathcal{P}$ is perfect. Hence Theorem 3 generalizes Corollary 3.6 in [2] about finite generation of iterated permutational wreath products ... $\imath_{X} \mathcal{P} l_{X} \mathcal{P}$.

Proposition 4. Let $G$ be a self-similar group of finite type given by patterns of depth $d$. If there exists $n \geq d$ such that the commutator of $\left.G\right|_{X^{[n]}}$ does not contain $\left.\operatorname{St}_{G}(n-1)\right|_{X^{[n]}}$ then the group $G$ is not finitely generated.

Proof. The proof uses the same arguments as in the first part of the proof above. Fix $n \geq d$ such that the commutator of $G_{n}:=\left.G\right|_{X^{[n]}}$ does not contain $\left.\operatorname{St}_{G}(n-1)\right|_{X^{[n]}}$. For every $k \in \mathbb{N}$ consider the map

$$
\varphi_{k}: G \rightarrow G_{n} /\left[G_{n}, G_{n}\right], \quad \varphi_{k}(g)=\left.\prod_{v \in X^{k}} g_{(v)}\right|_{X^{[n]}}\left[G_{n}, G_{n}\right]
$$

Since $G_{n} /\left[G_{n}, G_{n}\right]$ is abelian every map $\varphi_{k}$ is a homomorphism. Now for every $m \in \mathbb{N}$ consider the homomorphism $\varphi: G \rightarrow \prod_{k=1}^{m} G_{n} /\left[G_{n}, G_{n}\right], \varphi(g)=\left(\varphi_{k}(g)\right)_{k=1}^{m}$. For every $k$ and every pattern $\left.a \in \operatorname{St}_{G}(n-1)\right|_{X[n]}$ there exists $g$ in the rigid stabilizer $\operatorname{RiSt}_{G}(v)$ of a vertex $v \in X^{k}$ such that $\left.g_{(v)}\right|_{X^{[n]}}=a$, and thus $\varphi_{k}(g)=a$ and $\varphi_{i}(g)=e$ for $i<k$. Since $\left.\operatorname{St}_{G}(n-1)\right|_{X[n]} /\left[G_{n}, G_{n}\right]$ is a homomorphic image of $G_{n}$, it follows that the abelian group $\left.\prod_{k=1}^{m} \operatorname{St}_{G}(n-1)\right|_{X^{[n]}} /\left[G_{n}, G_{n}\right]$ is a homomorphic image of $G$ for every $m$. Hence $G$ is not finitely generated.

The next statement generalizes Proposition 2 in [10]. 
Corollary 5. Let $\mathcal{P}$ be an abelian pattern group. The group $G_{\mathcal{P}}$ is finitely generated if and only if it is finite.

Proof. The statement follows from Proposition 1 and Proposition 4 with $n=d$.

Corollary 6. Take a cyclic subgroup $C<\operatorname{Sym}(X)$ and consider the group $C 2_{X} C$ as a natural subgroup of Aut $X^{[2]} \cong \operatorname{Sym}(X){ }_{2} \operatorname{Sym}(X)$. Then for any nilpotent pattern group $\mathcal{P}<C 2_{X} C$ the group $G_{\mathcal{P}}$ is finitely generated if and only if it is finite.

Proof. Since $\mathcal{P} / \operatorname{St}_{\mathcal{P}}(1)$ is cyclic, the commutator $[\mathcal{P}, \mathcal{P}]$ is a subgroup of $\operatorname{St}_{\mathcal{P}}(1)$. If it is a proper subgroup then the group $G_{\mathcal{P}}$ is not finitely generated by Proposition 4 . Suppose $[\mathcal{P}, \mathcal{P}]=\operatorname{St}_{\mathcal{P}}(1)$. For any $a, b \in \mathcal{P}$ there exists $k \in \mathbb{N}$ such that $a^{k} b$ or $b^{k} a$ belongs to $\mathrm{St}_{\mathcal{P}}(1)$. Using the equality $[a, b]=\left[a, a^{k} b\right]=\left[b^{k} a, b\right]$ we obtain that $[\mathcal{P}, \mathcal{P}]=\left[\mathcal{P}, \operatorname{St}_{\mathcal{P}}(1)\right]$. Since $\mathcal{P}$ is nilpotent, the last equality implies that $[\mathcal{P}, \mathcal{P}]=\operatorname{St}_{\mathcal{P}}(1)=\{1\}$ and hence the group $G_{\mathcal{P}}$ is finite by Proposition 1 .

\section{A few classification results}

In this section we classify self-similar groups of finite type for the binary alphabet $X=$ $\{0,1\}$ and depth $\leq 4$. All computations were made in GAP. Our strategy for classifying self-similar groups of finite type of a given depth $d$ is the following. First we find all subgroups in Aut $X^{[d]}$, then minimize all subgroups and obtain the number of all minimal pattern groups, which is equal to the number of self-similar groups of finite type of a given depth as subgroups in Aut $X^{*}$. Further we distinguish all finite groups using Proposition 1 , Then we apply Proposition 4 for small values of $n$ to distinguish groups that are not finitely generated. An infinite self-similar group over the binary alphabet is level-transitive (see [3, Lemma 3]), hence the rest of the groups are level-transitive and we can apply Theorem 3 , In this way it was possible to obtain the following results.

Depth $d=2$. This case was treated in [10]. There are ten subgroups in Aut $X^{[2]}$, six minimal pattern subgroups, and hence six self-similar groups of finite type. Among them there are three finite groups, namely the trivial group and two groups isomorphic to $C_{2}$, and the other three groups are not finitely generated (Proposition 4 works with $n=2$ ).

Depth $d=3$. There are 576 subgroups in Aut $X^{[3]}, 60$ minimal pattern subgroups, and hence 60 self-similar groups of finite type. Among them there are 23 finite groups, namely the trivial group, two groups isomorphic to $C_{2}$, four groups isomorphic to $C_{2} \times C_{2}$, 16 groups isomorphic to the dihedral group $D_{8}$. The other 37 groups are not finitely generated (27 groups satisfy Proposition 4 with $n=3$ and 10 groups with $n=4$ ).

Corollary 7. A self-similar group of finite type given by patterns of depth $d \leq 3$ over the binary alphabet is either finite or not finitely generated.

Depth $d=4$. There are 4544 self-similar groups of finite type. Among them there are 1535 finite groups, namely the trivial group, two groups isomorphic to $C_{2}$, four groups isomorphic to $C_{2} \times C_{2}, 16$ groups isomorphic to $D_{8}$, eight groups isomorphic to $C_{2} \times C_{2} \times C_{2}$, 
96 groups isomorphic to $C_{2} \times D_{8}, 128$ groups isomorphic to $\left(C_{2} \times C_{2} \times C_{2} \times C_{2}\right) \rtimes C_{2}$, 256 groups isomorphic to $\left(\left(\left(C_{4} \times C_{2}\right) \rtimes C_{2}\right) \rtimes C_{2}\right) \rtimes C_{2}$, and 1024 groups isomorphic to Aut $X^{[3]} \cong C_{2}{ }_{X} C_{2}{ }_{X} C_{2}$. Among the rest of the groups there are 2977 not finitely generated (1235 groups satisfy Proposition 4 with $n=4,778$ groups with $n=5,508$ groups with $n=6,200$ groups with $n=7$, and 256 groups with $n=8$ ) and 32 finitely generated groups that satisfy Theorem 3 with $n=6$. The pattern groups of these 32 self-similar groups of finite type all have order 4096, their restriction on $X^{[3]}$ is equal to Aut $X^{[3]}$, and among them there are 20 pairwise non-isomorphic groups. These pattern groups can be described as follows. Let us consider the group Aut $X^{[4]}$ as a natural subgroup of the symmetric group $\operatorname{Sym}(16)$ on the set $\{1,2, \ldots, 16\} \leftrightarrow X^{4}$ and fix the permutations:

$$
\begin{aligned}
a_{1} & =(1,9)(2,10)(3,11)(4,12)(5,13)(6,14)(7,15)(8,16) \\
a_{2} & =(1,10,2,9)(3,11)(4,12)(5,14,6,13)(7,15)(8,16) \\
a_{3} & =(1,10)(2,9)(3,11)(4,12)(5,13)(6,14)(7,15)(8,16) \\
a_{4} & =(1,9,2,10)(3,11)(4,12)(5,14,6,13)(7,15)(8,16) \\
&
\end{aligned}
$$

Then the 32 pattern groups mentioned above is the family of groups $\mathcal{P}_{i j k}=\left\langle a_{i}, b_{j}, c_{k}\right\rangle$. In this family: the self-similar group of finite type $G_{\mathcal{P}_{123}}$ is the closure of the Grigorchuk group and $G_{\mathcal{P}_{111}}$ is the closure of the iterated monodromy group of $z^{2}+i[6]$.

\section{References}

[1] L. Bartholdi, R. Grigorchuk, Z. Šunik, Branch groups, in Handbook of algebra, Vol. 3 (North-Holland, Amsterdam, 2003), pp. 989-1112.

[2] I. Bondarenko, Finite generation of iterated wreath products, Arch. Math. 95(4) (2010) 301-308.

[3] I. Bondarenko, R. Grigorchuk, R. Kravchenko, Y. Muntyan, V. Nekrashevych, D. Savchuk, Z. Sunik, On classification of groups generated by 3 -state automata over a 2-letter alphabet, Algebra Discrete Math. (1) (2008) 1-163.

[4] R. I. Grigorchuk, Solved and unsolved problems around one group, in Infinite Groups: Geometric, Combinatorial and Dynamical Aspects, eds. L. Bartholdi, T. CeccheriniSilberstein, T. Smirnova-Nagnibeda, A. Zuk, Vol. 248 (Progress in Mathematics Series, 2005), pp. 117-218.

[5] R. I. Grigorchuk, Just infinite branch groups, in New horizons in pro-p groups, eds. M. Sautoy, D. Segal, A. Shalev (Birkhauser Boston, 2000), pp. 121-179.

[6] R. Grigorchuk, D. Savchuk, Z. Šunić, The spectral problem, substitutions and iterated monodromy, CRM Proceedings and Lecture Notes 42 (2007) 225-248. 
[7] D. Lind, B. Marcus, An Introduction to Symbolic Dynamics and Coding (Cambridge University Press, Cambridge, 1995).

[8] V. Nekrashevych, Self-similar groups, Mathematical Surveys and Monographs, Vol.117 (American Mathematical Society, Providence, 2005).

[9] Z. Šunić, Hausdorff dimension in a family of self-similar groups, Geom. Dedicata 124(1) (2007) 213-236.

[10] Z. Šunić, Pattern closure of groups of tree automorphisms, Bull. Math. Sci. 1 (2011) $115-127$. 